\title{
Quantitative Assessment of Methods Used To Obtain Rate Constants from Molecular Dynamics Simulations-Translocation of Cholesterol across Lipid Bilayers
}

Filipe, Hugo A. L.

2018-07

Filipe , H A L , Javanainen , M , Salvador , A , Galvao , A M , Vattulainen , I , Loura , L M S \& Moreno, M J 2018 , ' Quantitative Assessment of Methods Used To Obtain Rate Constants from Molecular Dynamics Simulations-Translocation of Cholesterol across Lipid Bilayers ' , Journal of Chemical Theory and Computation , vol. 14 , no. 7 , pp. 3840-3848 . https://doi.org/10.1021/acs.jctc.8b00

http://hdl.handle.net/10138/321826

https://doi.org/10.1021/acs.jctc.8b00150

acceptedVersion

Downloaded from Helda, University of Helsinki institutional repository.

This is an electronic reprint of the original article.

This reprint may differ from the original in pagination and typographic detail.

Please cite the original version. 


\title{
Quantitative assessment of methods used to obtain rate constants from molecular dynamics simulations - translocation of cholesterol across lipid bilayers
}

\author{
Hugo A. L. Filipe ${ }^{1,4}$, Matti Javanainen ${ }^{2,3}$, Armindo Salvador ${ }^{1,4}$, Adelino M. Galvão5, Ilpo Vattulainen ${ }^{3,2,6^{*}}$, Luís M. S. \\ Loura $^{1,7^{*}}$, and Maria João Moreno ${ }^{1,8^{*}}$ \\ 1 Coimbra Chemistry Center, University of Coimbra, P-3004-535 Coimbra (Portugal) \\ 2 Laboratory of Physics, Tampere University of Technology, FI-33101 Tampere (Finland) \\ 3 Department of Physics, University of Helsinki, FI-ooo14 University of Helsinki (Finland) \\ 4 CNC - Center for Neuroscience and Cell Biology, University of Coimbra, P-3004-517 Coimbra (Portugal) \\ 5 CQE - Centro de Química Estrutural, Instituto Superior Técnico, Universidade de Lisboa, Av. Rovisco Pais \\ 1049-001 Lisboa (Portugal) \\ 6 MEMPHYS - Center for Biomembrane Physics, www.memphys.dk \\ 7 Faculty of Pharmacy, University of Coimbra, P-30oo-548 Coimbra (Portugal) \\ 8 Chemistry Department, University of Coimbra, P-3004-535 Coimbra (Portugal)
}

KEYWORDS. Amphiphile flip-flop; Lipid Bilayers; Permeation; Molecular Dynamics simulations; Cholesterol.

\begin{abstract}
Accurately calculating rate constants of macroscopic chemical processes from molecular dynamics simulations is a long-sought but elusive goal. The problem is particularly relevant for processes occurring in biological systems, as is the case for ligand-protein and ligand-membrane interactions. Several formalisms to determine rate constants from easily accessible free energy profiles $\left[\Delta G^{\circ}(\mathrm{z})\right]$ of a molecule along a coordinate of interest have been proposed. However, their applicability for molecular interactions in condensed media has not been critically evaluated or validated. This work presents such evaluation and validation, and introduces improved methodology. As a case study, we have characterized quantitatively the rate of translocation of cholesterol across 1-palmitoyl-2-oleoyl-sn-glycero-3-phosphocholine bilayers. Translocation across lipid bilayers is the rate limiting step in the permeation of most drugs through biomembranes. We use coarsegrained molecular dynamics simulations and different kinetic formalisms to calculate this rate constant. A self-consistent test of the applicability of various available formalisms is provided by comparing their predictions with the translocation rates obtained from actual events observed in long unrestrained simulations. To this effect, a novel procedure was used to obtain the effective rate constant, based on an analysis of time intervals between transitions among different states along the reaction coordinate. While most tested formalisms lead to results in reasonable agreement (within a factor of 5) with this effective rate constant, the most adequate one is based on the explicit relaxation frequencies from the transition state in the forward- and backward-directions along the reaction coordinate.
\end{abstract}

Kinetics of molecular interactions is at the basis of all chemical processes. For systems out of equilibrium, it is often the kinetics of a specific event that determines the final outcome during the pertinent time scale. This is particularly relevant for drug distribution and disposition due to the many interactions established between the drug and the binding agents and barriers present on the way between the administration and the target sites. ${ }^{1-2}$ Once at the target site, drug efficacy is also affected by the kinetics of the interactions due to competition with additional ligands available and to the non-equilibrium properties of the biological systems. ${ }^{3-4}$ Knowing the rate at which these molecular interactions occur is a difficult task, however. Experimental determinations are feasible only when the interaction originates changes in a physical observable (spectroscopic, calorimetric) that can be measured in the time scale of the process..$^{-12}$ The ability to characterize these rates from molecular dynamics (MD) simulations would be a precious asset to assist the early steps of drug design and optimization. Important limitations include the adequacy of the force field employed,,$^{13-15}$ and the sensitivity of the ensemble, ergodicity and dynamical behavior of the simulated system to the choice of the thermostat ${ }^{16-17}$. While 
these are reasons for concern, there is active research on algorithm improvement and validation of MD simulations. ${ }^{18-19}$ In fact, MD is currently being used by several researchers to obtain information on the rate of molecular interactions. ${ }^{20-31}$

To quantitatively assess the methodologies available to extract rate constants from MD simulation data, a simple system should be used. While for protein-ligand interactions, the reaction path is not clear, permeation through biomembranes has an intuitive reaction coordinate - the direction normal to the lipid bilayer plane. This, together with the importance of permeation in drug distribution and disposition, led us to address the rate of passive permeation through biomembranes. This process involves several steps, namely association with the membrane leaflet in contact with the aqueous media, translocation into the opposite leaflet, and dissociation from the membrane into the aqueous compartment on the opposite side. To guarantee a significant solubility in the aqueous media, most drugs are relatively polar, and therefore translocation between membrane leaflets is usually the rate limiting step in permeation. ${ }^{22,32-33}$

In principle, the rate constant for a process can be straightforwardly computed from the frequency of events observed in the course of a MD simulation run. However, this strategy is impracticable for most processes because high energy barriers render those events exceedingly rare in the simulated timescale. ${ }^{28,34}$ This limitation can be circumvented by using strategies for enhanced sampling of improbable states. Among them, the most widely used is the Umbrella Sampling (US) ${ }^{35-36}$ method, which is applied to the calculation of Potential of Mean Force (PMF) profiles along a chosen reaction coordinate. In this context, the PMF represents the Gibbs free energy variation of the system as a function of the depth of the permeating molecule of interest within the lipid bilayer, from which the rate constant $(k)$ of the process may be calculated using an Arrhenius-like equation

$$
k=A e^{-\Delta^{\ddagger} G^{\circ} / R T}
$$

where $R$ is the gas constant, $T$ the absolute temperature, and $\Delta^{\ddagger} G^{\circ}$ is the activation Gibbs free energy (height of the energy barrier between the initial and transition states of the process under consideration). Although necessary, $\Delta^{\ddagger} G^{\circ}$ is not sufficient to calculate the rate constant. This calculation requires knowledge of the pre-exponential coefficient $(A)$. Several approaches have been proposed to calculate this pre-exponential coefficient, ${ }^{37-43}$ and some have been used to calculate rate constants from the PMFs obtained in MD simulations. ${ }^{28,30,44}$ However, a systematic and self-consistent evaluation of the distinct methods available has not been reported.

The quantitative evaluation of the adequacy of the distinct methods to calculate rate constants from energy profiles requires the use of a self-consistent approach where both the kinetic events and the free energy profiles are obtained for the same system and with the same constraints. The comparison between experimental results (rate constants) with predictions by the different methods based on energy profiles obtained from MD simulations is not a self- consistent approach, as it includes the eventual approximations and limitations associated with the force fields and with the methods used to sample improbable states. One possible approach is to use long enough unrestrained simulations to obtain the rate constants directly from kinetic events and the free energy profile from the density of the solute at the distinct locations in the reaction path. To obtain statistically significant results with reasonable computational cost, coarse grained (CG) simulations may be needed at this step.

This is the methodology followed in this work to characterize the rate of translocation of cholesterol (Chol) through 1-palmitoyl-2-oleoyl-sn-glycero-3-phosphocholine (POPC) bilayers. Besides the evident importance of cholesterol as a major component of plasma membranes, the choice of this solute ensures that a significant number of translocation (albeit no desorption) events may be observed in unrestrained CG MD simulations. ${ }^{27-31,34}$ In these simulations, the rate constant of translocation $\left(k_{\mathrm{f}}\right)$ was calculated from the distribution of time intervals between translocation events. In addition, the free energy variation at distinct depths in the lipid bilayer was obtained directly from the transverse density distribution of cholesterol. Biased US simulations have also been performed, and the PMF profiles obtained were compared to those from unrestrained simulations. The rate constant of translocation was then calculated from the PMFs using the distinct formalisms available and compared with that obtained directly using unrestrained MD simulations.

The results show that different formalisms lead to distinct values for the translocation rate constant. Among the methods that estimate $k$ from the free energy profile, the best accordance with the values determined directly from unrestrained simulations uses pre-exponential coefficients $A$ calculated using explicit frequencies for the relaxation of the solute from the position at the transition state, i.e. that of maximal free energy along the translocation path.

\section{Materials and Methods}

Unrestrained MD Simulations. MD simulations and analysis of trajectories was carried out using the GROMACS 5.0.x package. ${ }^{45}$ The topology of the POPC and Chol molecules consisted of a CG description from Martini force field..$^{31,} 4^{6}$ The starting structure - a fully hydrated POPC/Chol (9:1) bilayer with 520 POPC, 56 Chol and 7200 water beads - was built with the insane.py script to setup Martini bilayer systems. ${ }^{47}$ The standard nonpolarizable Martini water model was used..$^{46}$ A detailed description of the methodology followed is given in the Supporting Information (SI), section S1. ${ }^{48-50}$

Umbrella sampling Simulations. For the Umbrella sampling set of simulations, a smaller bilayer, consisting of 144 POPC, 16 Chol and 2182 water beads was constructed with insane.py. ${ }^{47}$ The distance from the Chol polar bead ${ }^{31}$ (here simply denoted as $\mathrm{OH}$ ) to the membrane center of mass (COM), projected to the membrane normal ( $z$ coordinate), was chosen as the reaction coordinate for solute permeation. To avoid local membrane perturbations, this COM definition was evaluated locally using the cylindrical pull geometry provided by GROMACS. ${ }^{21}$ The umbrella potential acts independently on the $\mathrm{OH}$ group of two Chol 
molecules, separated by $4 \mathrm{~nm}$. The adjacent umbrella windows spanned the space between the membrane center $(\mathrm{z}=\mathrm{o})$ into the bulk water region $(|\mathrm{z}|=4 \mathrm{~nm})$, separated by $0.1 \mathrm{~nm}$. After completing the simulations, the unbiased PMF was obtained using the weighted histogram analysis method. $^{21,51}$

Unrestrained relaxation from the US transition state. Several simulations where a Chol molecule is allowed to relax from the transition state identified by the US simulations, towards its equilibrium position, were performed. For this purpose, 100 frames, equally separated in time, were generated for the cases where each restrained Chol molecule was at $0.7 \mathrm{~nm}$ from the bilayer center. A total of 200 simulations, of 20 ns each, were performed.

Obtaining the rate constants from the time dependence of cholesterol position in the bilayer. The position of cholesterol in the lipid bilayer at a given simulation time was converted into a state, taking into account the Gibbs free energy profile obtained from the cholesterol density distribution in the bilayer. The distinct states considered are: i) equilibrium position in one leaflet (state a); ii) transition state (state b); iii) equilibrium position in the other leaflet (state c); and anywhere else (state $x$ ). The boundaries of each state correspond to the values of $z$ where the Gibbs free energy deviates $R T$ from the minimum (state a and c), or from the maximum (state b) in that state (cf. Figure 1 ). The $z$ coordinate at each time step was encoded as a character denoting the respective state, and the kinetic events identified through string pattern matching using Mathematica v. 10.4 ${ }^{52}$. The time at which cholesterol first entered the initial equilibrium state (a or c) and first entered the final equilibrium state (c or a, respectively) was registered, and the time interval $(\Delta t)$ was calculated. This is the time interval between translocation events (see Figures $\mathrm{S}_{2}$ and $\mathrm{S}_{3}$ for details). The rate constant was then calculated from the distribution of $\Delta t$ values for the event type considered. For this purpose, the total number of events was considered as the concentration of reactant at $t=0$, and one reactant is consumed at each event (defined by the $\Delta t$ values ordered from smaller to larger). The time decay of the reactant concentration (number of events) was analyzed by a single exponential function to obtain the rate constant. For some events, the data was not well described by the single exponential function and a double exponential was used. In this case, the rate constant considered was either the smallest or the weighted average of both (see below for further details).

The events corresponding to relaxation frequencies from the transition state were analyzed following the same approach, see SI section Si for the regular expressions used for pattern matching. In this case, $\Delta t$ was calculated as the time interval between entering the transition state (b) for the first time in the path until first entering the final equilibrium state (a or c).

The simulation time led to proper convergence of the calculated translocation rate constant values at all temperatures. As described below, the characteristic time for this process is $\sim 1 / k_{\mathrm{f}}=0.3 \mu \mathrm{s}$ at $310 \mathrm{~K}$, that is 30 -fold shorter than the simulation time. Even in the most unfavorable case,
$T=293 \mathrm{~K}$, the simulation time is still $>$ 10-fold the corresponding characteristic translocation time. Clear convergence is obtained well within the simulation time window, as illustrated by representing the recovered $k_{\mathrm{f}}$ value as a function of the time length used for analysis (Figure $S_{1}$ ).

Obtaining the rate constants from the free energy profile. The formalisms used to calculate the rate constant from the free energy barrier associated with the transformation $\left(\Delta^{\ddagger} G^{\circ}\right)$, are given in the SI section S2. The transition state theory (TST), ${ }^{37-39}$ explicit relaxation frequencies (ERFs), ${ }^{37},{ }^{2-43}$ diffusion through the top of the energy barrier (Diff), ${ }^{40-41}$ and the inhomogeneous solubility-diffusion model (ISDM), ${ }^{9}, 53-55$ were the formalisms considered.

\section{Results and discussion}

Rate constant from translocation events in unrestrained MD simulations. The translocation events were identified from the time dependence of the $z$ coordinate of each cholesterol molecule (see example for one cholesterol molecule in Figure S2), and the translocation rate constant obtained from the time distribution between events.

The movement between membrane leaflets was automatically followed through the assignment of the instantaneous location of cholesterol to either the equilibrium states, the transition state, or anywhere else. The definition of the distinct states was based on cholesterol Gibbs free energy profile $\Delta G^{\circ}(z)$, which was calculated from the density distribution $(d(z))$ of cholesterol along the bilayer normal $(z)$, Equation ( 2$)$, where $z_{\mathrm{Eq}}$ is the $z$ coordinate at the equilibrium position, leading to $\Delta G^{\circ}\left(z_{\mathrm{Eq}}\right)=0$.

$$
\Delta G^{\mathrm{o}}(z)=-R T \ln \left(d(z) / d\left(z_{\mathrm{Eq}}\right)\right)
$$

The density distribution of cholesterol COM and that of its $\mathrm{OH}$ group at all temperatures studied is shown in the SI (Figure $\mathrm{S}_{4}$ ). The resulting $\Delta G^{\circ}(\mathrm{z})$ at $310 \mathrm{~K}$ is shown in Figure 1 , with the identification of the distinct states.

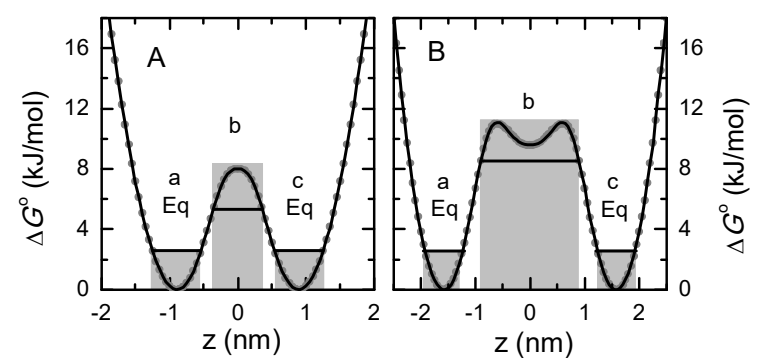

Figure 1 - Dependence of $\Delta G^{\circ}$ on the position of cholesterol COM (plot A) or OH group (plot B), with the identification of the 3 states considered for cholesterol localization: $a, b$ and $c$. The horizontal lines correspond to a variation in $\Delta G^{\circ}$ equal to $\mathrm{RT}$ relative to the local minima (Eq) or maxima (b).

The cholesterol COM tends to be located deeper in the membrane than the $\mathrm{OH}$ group, as expected from the preferred orientation of cholesterol in lipid bilayers, with the $\mathrm{OH}$ group pointing towards the bilayer/water interface. Furthermore, the probability density of the location of the cholesterol $\mathrm{OH}$ group presents a local maximum at the center of the membrane, whereas that of the COM presents a minimum therein. This distinct behavior of COM and 
$\mathrm{OH}$ densities at the center of the bilayer leads to different Gibbs free energy profiles (Figure 1). Given the inverse relation between the solute density and the corresponding Gibbs free energy (equation (2)), a minimum in the density leads to a maximum in $\Delta G^{\circ}$. The profile of $\Delta G^{\circ}(z)$ for cholesterol COM presents a local maximum at $z=0$, while that of the $\mathrm{OH}$ group has a local minimum at $z=0$, flanked by two local maxima at $\sim \mathbf{n m}$ from the center of the bilayer. The distinct states relevant for the translocation process were defined by the range of $z$ values for which $\Delta G^{\circ}(z)$ is within $R T$ of the corresponding minimum or maximum at that region. Therefore, the local minimum at $z=0$ for $\Delta G^{\circ}(\mathrm{z})$ obtained from the localization of cholesterol $\mathrm{OH}$ was not considered as an independent state, because it differs from the adjacent maxima by less than $R T$.

The time decay of the number of translocation events was used to obtain the rate constant of translocation (Figure 2). Very similar values were obtained for the rate of translocation in both directions. Therefore the rate constants considered thereafter were obtained from the analysis of the cumulative translocation events, and are referred as the "effective" rate constants below. The results obtained from the coordinates of cholesterol COM follow a bi-exponential function, with a very fast component ( $\tau \cong 2 \mathrm{~ns}$ ), and a slow component ( $\tau \cong 200 \mathrm{~ns}$ ). In contrast, the decay observed when the $\mathrm{OH}$ group is followed is well described by a single exponential function ( $\tau \cong 300 \mathrm{~ns}$ ). The rate constants for translocation at $310 \mathrm{~K}$ obtained from the unrestrained simulations are shown in Table I, and the results at the other temperatures are given in Table $\mathrm{S}$. The very fast component observed when cholesterol COM is considered corresponds to situations where cholesterol $\mathrm{COM}$ has reached the opposite leaflet but the $\mathrm{OH}$ group remained in the original leaflet. Those events correspond to ineffective translocation, as discussed in the last section of the SI. Accordingly, in this case, only the slow component was considered for the time decay of the translocation events.

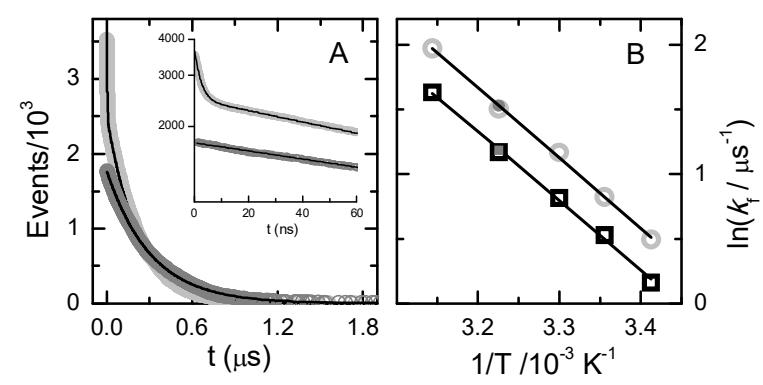

Figure 2 - A - Time dependence of the decay in the number of translocation events when following cholesterol COM (O)) or $\mathrm{OH}(\mathrm{O})$. The black lines are the best fit with a bi (COM) or mono $(\mathrm{OH})$ exponential function. The decay during the first $60 \mathrm{~ns}$ is represented in the inset as a semi-logarithmic plot to highlight deviations from a mono-exponential behavior. B Temperature dependence of the translocation rate constant obtained from the position of cholesterol COM $(\bigcirc)$ and $\mathrm{OH}$ group $(\square)$. A replicate experiment has been performed at 310 $\mathrm{K}$ which is represented by solid symbols in dark gray ( $\bullet$ or $\square$ ).

The values obtained for the rate constant of translocation (both from the movement of cholesterol $\mathrm{COM}$ or $\mathrm{OH}$ group) are in the same order of magnitude as those obtained previously by MD simulations. ${ }^{27-31}$. However, this should not be taken as a quantitative estimate of the rate constant of cholesterol translocation in real lipid membranes. The comparison with experimental data is precluded by the lack of an exact value due to the very fast translocation of cholesterol. ${ }^{56-57}$ A detailed analysis of cholesterol orientation near the translocation events (effective and ineffective) is presented in SI section $\mathrm{S}_{13}$, to obtain information regarding the mechanism of cholesterol translocation.

The rate constants for cholesterol translocation obtained at the distinct temperatures studied are shown in Figure $2 \mathrm{~B}$. The logarithm of the rate constants varied linearly with $1 / T$, leading to an enthalpy of activation for translocation $\left(\Delta^{\ddagger} H^{\circ}\right)$ equal to $45 \pm 1 \mathrm{~kJ} \mathrm{~mol}^{-1}$. The same value was obtained when translocation is reported by either cholesterol $\mathrm{COM}$ or its $\mathrm{OH}$ group. However, the intercept is larger for the case of cholesterol COM, reflecting the higher value obtained for the translocation rate constant. The difference in the intercepts gives a direct measure of the entropy difference between cholesterol translocation when considered from the movement of its $\mathrm{COM}$ or its $\mathrm{OH}$ group. The difference obtained $\left(9.6 \mathrm{JK}^{-1} \mathrm{~mol}^{-1}\right)$ corresponds to $3 \mathrm{~kJ} \mathrm{~mol}^{-1}$ at $310 \mathrm{~K}$, in good agreement with the $\Delta G^{\circ}(\mathrm{z})$ profiles shown in Figure 1.

The enthalpy of activation in translocation is much higher than the corresponding Gibbs free energy variation $\left(\Delta^{\ddagger} G^{\circ}\right)$, see Figure 1 for $310 \mathrm{~K}$ and Figure $\mathrm{S}_{4}$ for the other temperatures. This indicates that the entropy variation associated with the formation of the transition state in translocation is significant and favorable, reflecting a decrease in the order of the bilayer when cholesterol is located in its central region.

From the Gibbs free energy profiles at the different temperatures, the values of $\Delta H^{\circ}$ and $\Delta S^{\circ}$ at the distinct depths in the bilayer may be obtained. Those values are relative as they depend on the reference considered for $\Delta G^{\circ}(z)$. In keeping with the data shown above, $\Delta G^{\circ}(z)$ was taken as zero at the equilibrium location. The results are shown in Figure $\mathrm{S}_{5}$. The values obtained for $\Delta^{\ddagger} H^{\circ}$ are similar to those calculated from the temperature dependence of the translocation rate constant (Figure $2 \mathrm{~B}$ )

Validation of the distinct kinetic formalisms to calculate rate constants from the free energy barrier. Calculation of rate constants from direct observation of events is limited to fast or very fast processes, because a significant number of independent events must be observed, often implying impossibly long simulations. In this section, the distinct formalisms to calculate the rate constant of translocation from the Gibbs free energy profile (presented in the SI, Section S2), will be applied. This provides an internally consistent validation of the formalisms available. Those that provide the best estimation of the previously obtained "effective" rate constant will be used in the next section to calculate the rate constant from the PMFs obtained using biased US simulations s5-36 $^{36}$.

The transition state theory, Equations $\left(\mathrm{S}_{1}\right)$ and $\left(\mathrm{S}_{2}\right)$, leads to a rate constant for translocation equal to $100 \mathrm{~ns}^{-1}$ at $310 \mathrm{~K}$, when considering absence of spatial diffusion 
problems or recrossing events $(\kappa=1)$. This value should be taken as an upper-bound of the rate constant, achievable only in the Kramers cross-over region (see SI section S2). ${ }^{8}$ In the strongly coupled environment of translocation we are clearly in the over-damped coupling regime with high friction coefficients and, as so, the transmission coefficient $\kappa$ considered is expected to be several orders of magnitude larger than the observed value. The actual value of $\kappa$, accounting for spatial diffusion, can be estimated by the quotient between the observed relaxation frequency into products $\left(f_{\mathrm{f}}\right)$ and the TST pre-exponential $\left(A_{T S T}\right)$, equation $\left(\mathrm{S}_{3}\right)$.

Since the rate of product formation is affected by events that bring the molecule to its initial state, it is therefore necessary to characterize quantitatively not only the relaxation frequency into the products $\left(f_{\mathrm{f}}\right)$ but also to the reactants $\left(f_{\mathrm{r}}\right)$. This may be directly calculated from the simulations using a methodology similar to that followed to obtain the rate constant for translocation, as described in the Methods section, and Figure S6. These explicit relaxation frequencies (ERF in the following) provide an improved estimate of $A_{T S T}$ (given by $f_{\mathrm{f}}$ ), as well as an estimation of the fractional forward relaxation frequency $\left(\eta_{\mathrm{f}}\right)$, equation $\left(\mathrm{S}_{4}\right)$, to unbias the rate constants of the effects of recrossings and adiabatic relaxation to the initial state. The results obtained are presented in Figures 3 and $\mathrm{S}_{7}$.

The decay in the number of events from the transition state in the forward direction is well described by a singleexponential function leading to $f_{\mathrm{f}}$ equal to $0.38 \mathrm{~ns}^{-1}$, when cholesterol is followed by its $\mathrm{OH}$ group. On the other hand, in the backward direction the decay requires at least two exponentials to be accurately described, the slow component being similar to $f_{\mathrm{f}}$ and the additional component almost one order of magnitude faster. The temperature dependence of the ERFs is shown in Figures $3 \mathrm{~B}, \mathrm{~S} 12$ and Table $\mathrm{S}$, being similar for all ERFs (slope of $\cong 2.5 \times 10^{3} \mathrm{~K}$ ).

The rate constants for translocation at $310 \mathrm{~K}$ calculated from $\Delta^{\ddagger} G^{\circ}$ and the respective ERFs are shown in Table I, and the results at all temperatures are given in Table $S_{1}$ and Figure S8. For the cases where a biphasic behavior was observed (backward relaxation frequency for cholesterol $\mathrm{COM}$ and $\mathrm{OH}$ group), two values were considered for $f_{\mathrm{r}}$; i) one obtained by averaging the characteristic times of the slow and fast components weighted by the respective number of events, and ii) the slow component only. The calculated translocation rate constant is larger when using the slower component because the efficiency of the reaction increases. Thus, when the weighted average of $f_{\mathrm{r}}$ leads to a good estimation of $k_{\mathrm{f}}$ (case of COM), using the slow component leads to a poor result. On the other hand, if the weighted average of $f_{\mathrm{r}}$ results in an underestimation of $k_{\mathrm{f}}$ (case of $\mathrm{OH}$ ), the quality of the estimation improves when using only the slower component.

When the relaxation processes are non-exponential, it may be advantageous to calculate $\eta_{\mathrm{f}}$ directly from the time correlation functions for the relaxation from the transition state, Figures $3 \mathrm{~A}$ and $\mathrm{S}_{7}$. The coefficient thus obtained is time dependent, $\eta_{\mathrm{f}}(t)$, being smaller at short times and increasing towards a plateau which should be used when estimating rate constants (see SI sections S2 and S8 for a more detailed interpretation). ${ }^{42-43}$. For the case of cholesterol translocation, the plateau values obtained $(\cong 0.5)$ are very similar to those calculated from equation $\left(\mathrm{S}_{4}\right)$ considering only the slower component of the ERFs.
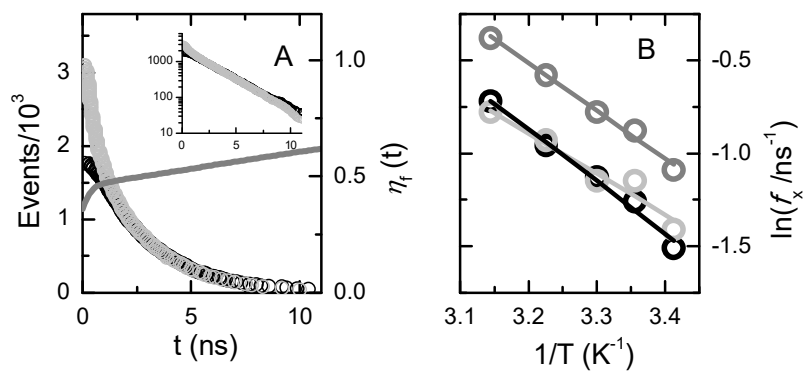

Figure 3 - Effective relaxation frequencies. Panel A: time dependence of the decay in the number of events for relaxation in the forward $(O)$ and backward $(\bigcirc)$ direction, when following cholesterol $\mathrm{OH}$, and corresponding fractional forward relaxation frequency $\eta_{\mathrm{f}}(t)(-)$. The decay is represented in the inset as a semi-logarithmic plot to highlight deviations from a mono-exponential behavior. Panel $\mathrm{B}$ : Temperature dependence of $f_{\mathrm{f}}(\mathrm{O})$, and the weighted average of the two exponentials $(\bigcirc)$ or the slow component $(\bigcirc)$ of $f_{\mathrm{r}}$.

Table I - Translocation rate constants obtained through the use of the distinct methods, and relevant parameters considered in the calculations, at $310 \mathrm{~K}$.

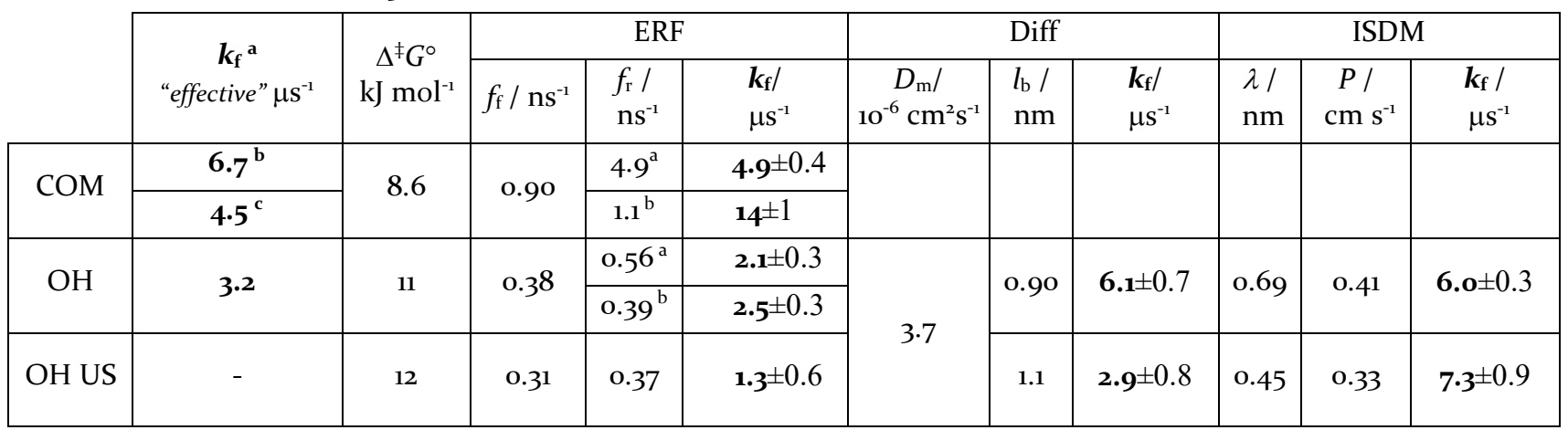

${ }^{\mathrm{a}}$ fitting standard errors $\leq 0.1 \%$, with $\mathrm{R}^{2} \geq 0.9998$ in all cases, ${ }^{\mathrm{b}}$ weighted average, ${ }^{\mathrm{c}}$ slower component. 
The most consensual choice for the reaction coordinate of a translocation process is the distance of the molecule's most polar group, in this case the $\mathrm{OH}$ group, to the center of the bilayer. In addition, when following translocation through the movement of the $\mathrm{OH}$ group, $\eta_{\mathrm{f}}$ is less time dependent and the plateau is approached at shorter times when compared to the overall relaxation from the transition state. Altogether, the best choice in this case seems to be the conjugation of the $\mathrm{OH}$ free energy profile with the slower component of the relaxation frequencies, which is equivalent to considering $\eta_{\mathrm{f}}$ near $1 / 2$. This is also the methodology that leads to rate constants in better agreement with those obtained directly from the translocation events in the unrestrained simulations. Unless stated otherwise, the following discussion will only refer to data obtained from the analysis of the position of cholesterol $\mathrm{OH}$ group.

An alternative procedure to calculate the pre-exponential in Equation (1) is to consider that it is limited by the diffusion of the solute through the high energy barrier that corresponds to the transition state, Equation $\left(\mathrm{S}_{5}\right)$. The width of the barrier is directly obtained from the Gibbs free energy profiles, Figures 1 and $\mathrm{S}_{4}$, being $0.9 \mathrm{~nm}$ for the position of cholesterol $\mathrm{OH}$ group at $310 \mathrm{~K}$.

The calculation of the diffusion coefficient in the $z$ direction $\left(D_{z}\right)$, can be done using several methods. ${ }^{54}, 59$ To the exclusive consideration of data from unrestrained simulations, the Einstein equation could be used. However, to maintain a local position of the Chol molecule very short time intervals must be considered, which, in addition to the intrinsic heterogeneity of the lipid bilayer in the $z$ direction, contributes to a nonlinear mean square displacement of cholesterol. Therefore, our choice for the calculation of $D_{z}(z)$ profiles, was through the analysis of the position autocorrelation function (PACF) of the data obtained from $\mathrm{Chol} \mathrm{OH}$ group in US simulations, as described in reference $^{59}$. The results obtained are presented in Figure $4 \mathrm{~A}$. The $D_{z}(z)$ profile is noisy and the rationalization of its dependence on $z$ is not clear. The average value of $D_{z}$ within the region of the energy barrier, $D_{\mathrm{m}}$, is $3.7 \times 10^{-6} \mathrm{~cm}^{2} \mathrm{~s}^{-1}$. This value was used in equation $\left(\mathrm{S}_{5}\right)$ to calculate the rate constant of translocation shown in Table I.

The rate constants of translocation calculated using this methodology are in good agreement (higher by a factor of 2) with those obtained from the direct analysis of translocation events ("effective" in table I).

Another approach to calculate rate constants from $\Delta G^{\circ}(z)$ and $D_{z}$, is the so-called inhomogeneous solubilitydiffusion model,53-54, 59 equations (S6) and (S7). The values for the permeation resistance through the membrane, $R(\mathrm{z})$, at $310 \mathrm{~K}$ are shown in Figure $4 \mathrm{~A}$. The resistance to the movement of cholesterol through the bilayer decreases from the region at the bilayer/water interface until $z_{\mathrm{Eq}}$, and then increases as cholesterol $\mathrm{OH}$ group approaches the center of the bilayer. The $R(z)$ profile is similar to that of $\Delta G^{\circ}(z)$ due to its exponential dependence on this parameter, while it depends only linearly on $D_{z}$. The integration of $R(z)$ between $\mathrm{z}_{\mathrm{Eq}}$ on opposite bilayer leaflets gives $P=0.41$ $\mathrm{cm} \mathrm{s}^{-1}$. The corresponding rate constant of translocation at $310 \mathrm{~K}$ calculated using equation $\left(\mathrm{S}_{7}\right)$, is $6.0 \mathrm{\mu s}^{-1}$; also in good agreement with the "effective" values. However, the temperature dependence obtained from this model is much lower than that obtained from the direct observation of the translocation events. Therefore, at the lower temperature studied this method significantly overestimates the rate constant of translocation.

The parameter $\lambda$ used in equation $\left(\mathrm{S}_{7}\right)$ converts the concentration of solute from units of moles per volume into moles per area. It should therefore reflect the mobility of the solute in the reaction coordinate while at the equilibrium position. Accordingly, we have used the difference between the two $z$ values where $\Delta G^{\circ}(z)=\Delta G^{\circ}\left(z_{\mathrm{Eq}}\right)+R T$. The values obtained at $310 \mathrm{~K}$ are given in Table I (Table $\mathrm{S} \mathbf{1}$ for all temperatures), being equal to $0.69 \mathrm{~nm}$ for cholesterol $\mathrm{OH}$ group. If a larger value were used (namely the thickness of the bilayer leaflet, $\cong 2 \mathrm{~nm}$ ) the calculated rate constant would be proportionally smaller.

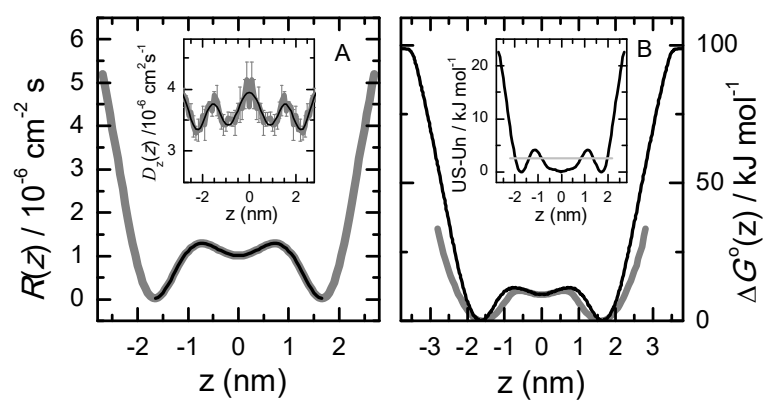

Figure 4 - A - Profile of $R(\mathrm{z})$ for the movement of cholesterol $\mathrm{OH}$ across the lipid bilayer $(-)$ and in the region considered in the calculation of $P(-)$ between $z_{\mathrm{Eq}}$ in the two leaflets. The inset shows $D_{z}(z)$ used in the calculation of $R(z)$. B - Profile of $\Delta G^{\circ}(z)$, for Unrestrained (-) and Umbrella Sampling (-) simulations. The difference between the $\Delta G^{\circ}(z)$ obtained with US and unrestrained (Un) simulations is shown in the inset, where the horizontal gray line corresponds to a difference equal to $R T$.

A comparison between the rate constants of translocation obtained by the distinct methods (except from the transition state theory with $\kappa=1$ ) is shown in Figure S8 for all temperatures studied. All methods lead to estimates of $k_{\mathrm{f}}$ within a factor of 5 from the "effective" value (yellow shaded regions). The best predictions lead to estimates of $k_{\mathrm{f}}$ within a factor of two (shaded region in green) and are obtained using the transition state theory with the pre-exponential coefficient calculated from the effective relaxation frequencies, equation $\left(\mathrm{S}_{4}\right)$.

Rate constants of translocation calculated from the free energy barrier obtained with Umbrella Sampling simulations. The free energy profile along a reaction coordinate may be obtained using the US approach ${ }^{35-36}$, with relatively low computational costs. Here, the $\mathrm{OH}$ group has been restrained at distinct $z$ values across the bilayer.

With the US simulations the whole system was sampled, from cholesterol in the aqueous media on one side of the bilayer $(z=4 \mathrm{~nm})$ towards the aqueous media on the opposite side $(\mathrm{z}=-4 \mathrm{~nm})$. The $\Delta G^{\circ}(\mathrm{z})$ profile is shown in Figure $4 \mathrm{~B}$, and compared to that obtained using unrestrained simulations. $\Delta G^{\circ}(z)$ also shows a local energy minimum when the $\mathrm{OH}$ group is located at $z=0$, flanked by local 
maxima at around $0.7 \mathrm{~nm}\left(z_{\ddagger}\right)$ and energy minima at $1.7 \mathrm{~nm}$ $\left(z_{\mathrm{Eq}}\right)$. Both $z_{\ddagger}$ and $z_{\mathrm{Eq}}$ are slightly shifted away from the bilayer center as compared with that observed in the unrestrained simulations. However, the major differences observed occur at values of $z$ above $z_{\mathrm{Eq}}$, where $\Delta G^{\circ}(z)$ increases more abruptly in the US simulations.

In this approach, the relaxation frequencies from the translocation transition state at $310 \mathrm{~K}$ were obtained from 20 ns unrestrained simulation, whose starting configurations were taken from the US simulations where the $\mathrm{OH}$ group of a cholesterol molecule had been restrained at $z_{\ddagger}$ (in both leaflets). The time evolution of cholesterol $\mathrm{OH}$ localization is shown in Figures S9 and Sio for some representative simulations started at $z_{\ddagger}=0.7 \mathrm{~nm}$. The behavior observed varied significantly with some molecules moving fast and directly into the nearest equilibrium position while others stay for a few ns in the high energy region and some eventually move directly into the equilibrium position in the opposite leaflet.

The relaxation frequencies were obtained from the analysis of the time intervals required for the OH group of cholesterol to enter in the $\mathrm{z}$ positions within $R T$ of one of the equilibrium positions. When the equilibrium position attained is on the same leaflet as the starting position, those time intervals define the relaxation frequency in the backward direction $\left(f_{\mathrm{r}}\right)$, whereas when the equilibrium position is on the opposite leaflet they define the forward relaxation frequency $\left(f_{\mathrm{f}}\right)$, see SI section Sio for further details. The relaxation frequencies obtained at $310 \mathrm{~K}$ are similar to those obtained for the movement of cholesterol $\mathrm{OH}$ group in fully unrestrained simulations. The values considered for $f_{\mathrm{r}}$ and $f_{\mathrm{f}}$ at the additional temperatures were obtained from the result at $310 \mathrm{~K}$ assuming the same temperature dependence as observed for the corresponding parameter in the unrestrained simulations (see SI, Figure S12).

The data from US simulations was used to estimate the rate constant of cholesterol translocation using the best models described in the previous section, the results obtained are shown in Figure 5. The method based on the explicit relaxation frequencies (ERF), equations (1) and $\left(\mathrm{S}_{4}\right)$, considering the slow component of the ERF in the backward direction $\left(f_{\mathrm{r}}\right)$, slightly underestimates (roughly by a factor of 4) the "effective" rate constant of translocation. This is due to the somewhat higher energy barrier and the slightly slower relaxation frequencies in the forward direction, $f_{\mathrm{f}}$, obtained in the US simulations (red symbols in Figure 5).

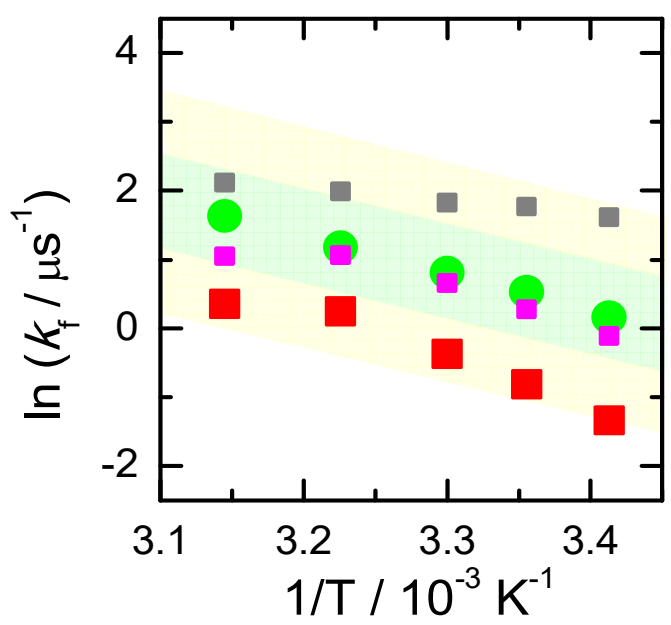

Figure 5 - Rate constants of cholesterol translocation directly from cholesterol movement between leaflets in unrestrained simulations (O); and calculated from the PMF obtained in US simulations restraining cholesterol $\mathrm{OH}$ group, with distinct kinetic formalism: ERF, using the slow component for the relaxation in the backward direction $(\square)$; Diff ( $\square)$; and ISDM ( $\square$ ). The green shaded regions represents deviations from the "effective" rate constant by a factor of 2 (green) or by a factor of 5 (yellow). Error bars are smaller than symbol size.

The rate constants of translocation calculated using the method of diffusion through the barrier (Diff), equation (1) and $\left(\mathrm{S}_{5}\right)$ is also shown in Figure 5, and lead to predictions very close to the values of the "effective" rate constants. Good predictions are obtained when using the inhomogeneous solubility-diffusion model (ISDM, squares in gray) at high temperatures. However, the temperature dependence is much weaker than that of the "effective" rate constants, leading to a poor agreement at low temperatures.

All three methods lead to estimates within a factor of 5 from the "effective" rate constant. In most cases the error indicated in Table 1 and $S_{1}$ reveal statistically significant differences from the effective rate constant, and among methods. However, those errors represent the uncertainties associated to the parameter estimation, and do not propagate the considerable uncertainties associated to the methods' underlying parameters. Therefore, the extent to which the discrepancies above reflect mostly these uncertainties or the different approximations and assumptions underlying each method is not at present clear. Given the large uncertainty associated with the calculation of the diffusion coefficient from US simulations, the use of the free energy barrier together with the effective relaxation frequencies provides possibly the most reliable method for the calculation of rate constants from US simulations.

\section{Concluding remarks}

This work presents the first self-consistent validation of the available formalisms for the calculation of rate constants from free energy profiles. The predictions from the formalisms were calibrated against the "effective" $k_{\mathrm{f}}$ values obtained from a large number of full translocation events observed in unrestrained CG MD simulations. 
The choice of the reaction coordinate is of major importance. For amphiphiles with a clearly localized polar group, the transverse position of the latter should be used as reaction coordinate for translocation, rather than its COM.

We showed that the direct calculation of rate constants from observed events in long unrestrained simulations is feasible and sound. However, it is prohibitively costly for solutes with high translocation barriers or for atomistic MD simulations. However PMF profiles may be rapidly obtained for these cases using biased simulations. Therefore, we evaluated alternative approaches to calculate the rate constant from free energy barriers. The method based on ERF calculation produces values that are in good agreement with the "effective" rate constants both for unrestrained and for biased (US) simulations. Moreover, it does not require the calculation of depth-dependent transverse diffusion coefficients $D_{z}(z)$, which are an important source of uncertainty. For the case of biased simulations, the calculation of the ERFs requires additional simulations. As shown by our study, for the calculation of ERFs, very short $(\sim 20 \mathrm{~ns})$ relaxation simulations starting at the transition state may be used in low order membranes. Although a large number of such runs must be carried out for proper statistics, the resulting computational effort is still very reasonable for $\mathrm{CG} \mathrm{MD}$, and even attainable for atomistic simulations.

This work shows that it is possible to accurately calculate rate constants using free energy profiles obtained from MD simulations. We are currently applying the methods developed herein to solutes for which reliable experimental data is available, as an external calibration required to use MD simulations to predict rate constants.

\section{Acknowledgment}

This work was partially supported by the Portuguese "Fundação para a Ciência e a Tecnologia" (FCT) through projects $007630 \quad$ UID/QUI/o0313/2013 and PT2020_PTDC_DTP-FTO_2784_2014, co-funded by COMPETE2020-UE. M.J. and I.V. thank the Academy of Finland (Centre of Excellence program) and the European Research Council (Advanced Grant CROWDED-PROLIPIDS) for financial support.

\section{Supporting information}

The Supporting Information is available free of charge on the ACS Publication website at DOI: 10.1021/acs.jctc.XXXXX.

This document contains a detailed description of the methods $\left(\mathrm{S}_{1}\right)$, including the kinetic formalisms ( $\left.\mathrm{S}_{2}\right)$; illustration of the translocation events and criteria used to identify them ( $\mathrm{S}_{3}$ and $\left.\mathrm{S}_{4}\right)$; results obtained from unrestrained and US simulations at all temperatures $\left(\mathrm{S}_{5}\right.$ and S6); criteria used and time correlation functions for the relaxation from the transition state in fully unrestrained simulations ( $\mathrm{S}_{7}$ and S8); self-consistent evaluation of the kinetic formalisms at all temperatures (S9); criteria used, time correlation functions, and frequencies, for the relaxation from the transition state in unrestrained simulations after US simulations (S1o and S11); overall results obtained for the kinetic parameters at all temperatures ( $\left.\mathrm{S}_{12}\right)$; and mechanistic look at cholesterol translocation $\left(\mathrm{S}_{13}\right)$.

\section{References}

1. Smith, D.; Artursson, P.; Avdeef, A.; Di, L.; Ecker, G. F.; Faller, B.; Houston, J. B.; Kansy, M.; Kerns, E. H.; Kramer, S. D.; Lennernas, H.; van de Waterbeemd, H.; Sugano, K.; Testa, B., Passive Lipoidal Diffusion and Carrier-Mediated Cell Uptake Are Both Important Mechanisms of Membrane Permeation in Drug Disposition. Mol. Phar, 2014, 11, 1727-1738.

2. Abbott, N. J., Blood-brain barrier structure and function and the challenges for CNS drug delivery. Journal of Inherited Metabolic Disease 2013, 36, 437-449.

3. Copeland, R. A.; Pompliano, D. L.; Meek, T. D., Opinion Drug-target residence time and its implications for lead optimization. Nature Reviews Drug Discovery 2006, 5, 730-739.

4. Swinney, D. C., Biochemical mechanisms of drug action: what does it take for success? Nature Reviews Drug Discovery 2004, 3 , 801-808.

5. Di Trani, J. M.; De Cesco, S.; O'Leary, R.; Plescia, J.; do Nascimento, C. J.; Moitessier, N.; Mittermaier, A. K., Rapid measurement of inhibitor binding kinetics by isothermal titration calorimetry. Nat. Commun. 2018, 9 .

6. Trigo-Mourino, P.; Griesinger, C.; Lee, D., Label-free NMRbased dissociation kinetics determination. Journal of Biomolecular Nmr 2017, 69, 229-235.

7. Hermann, K. F.; Neuhaus, C. S.; Micallef, V.; Wagner, B.; Hatibovic, M.; Aschmann, H. E.; Paech, F.; Alvarez-Sanchez, R.; Kramer, S. D.; Belli, S., Kinetics of lipid bilayer permeation of a series of ionisable drugs and their correlation with human transporter-independent intestinal permeability. European Journal of Pharmaceutical Sciences 2017, 104, 150-161.

8. Eyer, K.; Paech, F.; Schuler, F.; Kuhn, P.; Kissner, R.; Belli, S.; Dittrich, P. S.; Kramer, S. D., A liposomal fluorescence assay to study permeation kinetics of drug-like weak bases across the lipid bilayer. J. Controlled Release 2014, 173, 102-109.

9. Martins, P. T.; Velazquez-Campoy, A.; Vaz, W. L. C.; Cardoso, R. M. S.; Valerio, J.; Moreno, M. J., Kinetics and Thermodynamics of Chlorpromazine Interaction with Lipid Bilayers: Effect of Charge and Cholesterol. J. Am. Chem. Soc. 2012, 134, 4184-4195.

10. Cardoso, R. M. S.; Martins, P. A. T.; Gomes, F.; Doktorovova, S.; Vaz, W. L. C.; Moreno, M. J., Chain-Length Dependence of Insertion, Desorption, and Translocation of a Homologous Series of 7-Nitrobenz-2-oxa-1,3-diazol-4-yl-Labeled Aliphatic Amines in Membranes. J. Phys. Chem. B 2011, 115, 10098-10108.

11. Moreno, M. J.; Estronca, L. M. B. B.; Vaz, W. L. C., Translocation of phospholipids and dithionite permeability in liquid-ordered and liquid-disordered membranes. Biophys. J. 2006, 91, 873-881.

12. McIntyre, J. C.; Sleight, R. G., Fluorescence Assay for Phospholipid Membrane Asymmetry. Biochemistry 1991, 30, 11819-11827.

13. Monticelli, L.; Tieleman, D. P., Force Fields for Classical Molecular Dynamics. In Biomolecular Simulations: Methods and Protocols, Monticelli, L.; Salonen, E., Eds. Humana Press: Totowa, NJ, 2013; Vol. 924, pp 197-213.

14. Lopes, P. E. M.; Guvench, O.; MacKerell, A. D., Current States of Protein Force Fields for Molecular Dynamics Simulations. In Molecular Modeling of Proteins, Kukol, A., Ed. Humana Press: New York, 2015; Vol. 1215, pp 47-71.

15. Lyubartsev, A. P.; Rabinovich, A. L., Force Field Development for Lipid Membrane Simulations. Biochimica et Biophysica Acta (BBA) - Biomembranes 2016, 1858, 2483-2497. 
16. Cho, K.; Joannopoulos, J. D., Ergodicity and dynamical properties of constant-temperature molecular dynamics. Phys. Rev. A 1992, 45, 7089-7097.

17. Hunenberger, P. H., Thermostat Algorithms for Molecular Dynamics Simulations. In Advanced Computer Simulation. Advances in Polymer Science, Holm, C.; Kremer, K., Eds. Springer: Berlin, Heidelberg, 2005; Vol. 173, pp 105-149.

18. Van Gunsteren, W. F.; Daura, X.; Hansen, N.; Mark, A. E.; Oostenbrink, C.; Riniker, S.; Smith, L. J., Validation of Molecular Simulation: An Overview of Issues. Angewandte Chemie 2018, 57 , 884-902.

19. Wong-Ekkabut, J.; Karttunen, M., The good, the bad and the user in soft matter simulations. Biochimica et Biophysica Acta (BBA) - Biomembranes 2016, 1858, 2529-2538.

20. Parisio, G.; Sperotto, M. M.; Ferrarini, A., Flip-Flop of Steroids in Phospholipid Bilayers: Effects of the Chemical Structure on Transbilayer Diffusion. J. Am. Chem. Soc. 2012, 134, 12198-12208.

21. Filipe, H. A. L.; Moreno, M. J.; Rog, T.; Vattulainen, I.; Loura, L. M. S., How To Tackle the Issues in Free Energy Simulations of Long Amphiphiles Interacting with Lipid Membranes: Convergence and Local Membrane Deformations. $J$. Phys. Chem. B 2014, 118, 3572-3581.

22. Filipe, H. A. L.; Salvador, A.; Silvestre, J. M.; Vaz, W. L. C.; Moreno, M. J., Beyond Overton's Rule: Quantitative Modeling of Passive Permeation through Tight Cell Monolayers. Mol. Phar, 2014, 11, 3696-3706.

23. Dickson, C. J.; Hornak, V.; Pearlstein, R. A.; Duca, J. S., Structure-Kinetic Relationships of Passive Membrane Permeation from Multiscale Modeling. J. Am. Chem. Soc. 2017, 139, 442-452.

24. Mollica, L.; Decherchi, S.; Zia, S. R.; Gaspari, R.; Cavalli, A.; Rocchia, W., Kinetics of protein-ligand unbinding via smoothed potential molecular dynamics simulations. Scientific Reports 2015, 5 .

25. Rydzewski, J.; Nowak, W., Ligand diffusion in proteins via enhanced sampling in molecular dynamics. Phys. Life Rev. 2017, 22-23, 58-74

26. Lotz, S. D.; Dickson, A., Unbiased Molecular Dynamics of 11 min Timescale Drug Unbinding Reveals Transition State Stabilizing Interactions. J. Am. Chem. Soc. 2018, 140, 618-628.

27. Choubey, A.; Kalia, R. K.; Malmstadt, N.; Nakano, A.; Vashishta, P., Cholesterol Translocation in a Phospholipid Membrane. Biophys. J. 2013, 104, 2429-2436.

28. Bennett, W. F. D.; Tieleman, D. P., Molecular simulation of rapid translocation of cholesterol, diacylglycerol, and ceramide in model raft and nonraft membranes. J. Lipid Res. 2012, 53, 421-429.

29. Jo, S.; Rui, H.; Lim, J. B.; Klauda, J. B.; Im, W., Cholesterol Flip-Flop: Insights from Free Energy Simulation Studies. J. Phys. Chem. B 2010, 114, 13342-13348.

30. Bennett, W. F. D.; MacCallum, J. L.; Hinner, M. J.; Marrink, S. J.; Tieleman, D. P., Molecular View of Cholesterol Flip-Flop and Chemical Potential in Different Membrane Environments. J. Am. Chem. Soc. 2009, 131, 12714-12720.

31. Marrink, S. J.; de Vries, A. H.; Harroun, T. A.; Katsaras, J.; Wassall, S. R., Cholesterol Shows Preference for the Interior of Polyunsaturated Lipid Membranes. J. Am. Chem. Soc. 2008, 130, $10-11$.

32. Testa, B.; Waterbeemb, G. F.; Guy, R., Pharmacokinetic Optimization in Drug Research. WILEY-VCH: Weinheim, 2001.

33. Thomae, A. V.; Koch, T.; Panse, C.; Wunderli-Allenspach, H.; Kramer, S. D., Comparing the lipid membrane affinity and permeation of drug-like acids: The intriguing effects of cholesterol and charged lipids. Pharm. Res. 2007, 24, 1457-1472.

34. Javanainen, M.; Martinez-Seara, H.; Vattulainen, I., Nanoscale Membrane Domain Formation Driven by Cholesterol. Scientific Reports 2017, 7, 1143.

35. Torrie, G. M.; Valleau, J. P., Nonphysical sampling distributions in Monte Carlo free-energy estimation: Umbrella sampling. J. Comput. Phys. 1977, 23, 187-199.
36. Kästner, J., Umbrella sampling. Wiley Interdiscip. Rev.: Comput. Mol. Sci. 2011, 1, 932-942.

37. Evans, M. G.; Polanyi, M., Some applications of the transition state method to the calculation of reaction velocities, especially in solution. Trans. Faraday Soc. 1935, 31, 0875-0893.

38. Eyring, H., The Activated Complex and the Absolute Rate of Chemical Reactions. Chemical Reviews 1935, 17, 65-77.

39. Wynne-Jones, W. F. K.; Eyring, H., The Absolute Rate of Reactions in Condensed Phases. The Journal of Chemical Physics 1935, 3, 492-502.

40. Kramers, H. A., Brownian motion in a field of force and the diffusion model of chemical reactions. Physica 1940, 7, 284-304.

41. aniansson, E. A. G., On The Rate of Many-Step Processes. In Chemical and Biological Applications of Relaxation Spectrometry, E., W.-J., Ed. D. Reidel: Dordrecht, 1975; Vol. 18, pp 245-253.

42. Chandler, D., Roles of Classical Dynamics and Quantum Dynamics on Activated Processes Occurring in Liquids. Journal of Statistical Physics 1986, 42, 49-67.

43. Straub, J. E.; Berne, B. J., A Statistical-Theory for the Effect of Nonadiabatic Transitions on Activated Processes. J. Chem. Phys. 1987, 87, 6111-6116.

44. Neuvonen, M.; Manna, M.; Mokkila, S.; Javanainen, M.; Rog, T.; Liu, Z.; Bittman, R.; Vattulainen, I.; Ikonen, E., Enzymatic Oxidation of Cholesterol: Properties and Functional Effects of Cholestenone in Cell Membranes. Plos One 2014, 9.

45. Abraham, M. J.; Murtola, T.; Schulz, R.; Páll, S.; Smith, J. C.; Hess, B.; Lindahl, E., GROMACS: High performance molecular simulations through multi-level parallelism from laptops to supercomputers. SoftwareX 2015, 1-2, 19-25.

46. Marrink, S. J.; Risselada, H. J.; Yefimov, S.; Tieleman, D. P.; de Vries, A. H., The MARTINI force field: Coarse grained model for biomolecular simulations. J. Phys. Chem. B 2007, 111, 7812-7824.

47. Wassenaar, T. A.; Ingólfsson, H. I.; Böckmann, R. A.; Tieleman, D. P.; Marrink, S. J., Computational Lipidomics with insane: A Versatile Tool for Generating Custom Membranes for Molecular Simulations. J. Chem. Theory Comput. 2015, 11, 21442155.

48. Bussi, G.; Donadio, D.; Parrinello, M., Canonical sampling through velocity rescaling. J. Chem. Phys. 2007, 126, 014101.

49. Berendsen, H. J. C.; Postma, J. P. M.; van Gunsteren, W. F.; DiNola, A.; Haak, J. R., Molecular dynamics with coupling to an external bath. J. Chem. Phys. 1984, 81, 3684-3690.

50. Humphrey, W.; Dalke, A.; Schulten, K., VMD: Visual molecular dynamics. Journal of Molecular Graphics 1996, 14, 33 38.

51. Hub, J. S.; de Groot, B. L.; van der Spoel, D., g wham-A Free Weighted Histogram Analysis Implementation Including Robust Error and Autocorrelation Estimates. Journal of Chemical Theory and Computation 2010, 6, 3713-3720.

52. Wolfram Research, I. Mathematica (Version 10), Wolfram Research, Inc.: Champaign, Illinois, 2015.

53. Diamond, J. M.; Katz, Y., Interpretation of Nonelectrolyte Partition-Coefficients Between Dimyristoyl Lecithin and Water. $J$. Membrane Biol. 1974, 17, 121-154.

54. Marrink, S. J.; Berendsen, H. J. C., Simulation of Water Transport Through a Lipid-Membrane. J. Phys. Chem. 1994, 98 , 4155-4168

55. Bemporad, D.; Essex, J. W.; Luttmann, C., Permeation of small molecules through a lipid bilayer: A computer simulation study. J. Phys. Chem. B 2004, 108, 4875-4884.

56. Hamilton, J. A., Fast flip-flop of cholesterol and fatty acids in membranes: implications for membrane transport proteins. Current opinion in lipidology 2003, 14, 263-271.

57. Steck, Theodore L.; Lange, Y., How Slow Is the Transbilayer Diffusion (Flip-Flop) of Cholesterol? Biophys. J. 2012, 102, 945-946. 
58. Pollak, E.; Grabert, H.; Hanggi, P., Theory of Activated Rate-Processes for Arbitrary Frequency-Dependent Friction Solution of the Turnover Problem. J. Chem. Phys. 1989, 91, 40734087.
59. Awoonor-Williams, E.; Rowley, C. N., Molecular simulation of nonfacilitated membrane permeation. Biochim. Biophys. Acta-Biomembr. 2016, 1858, 1672-1687. 

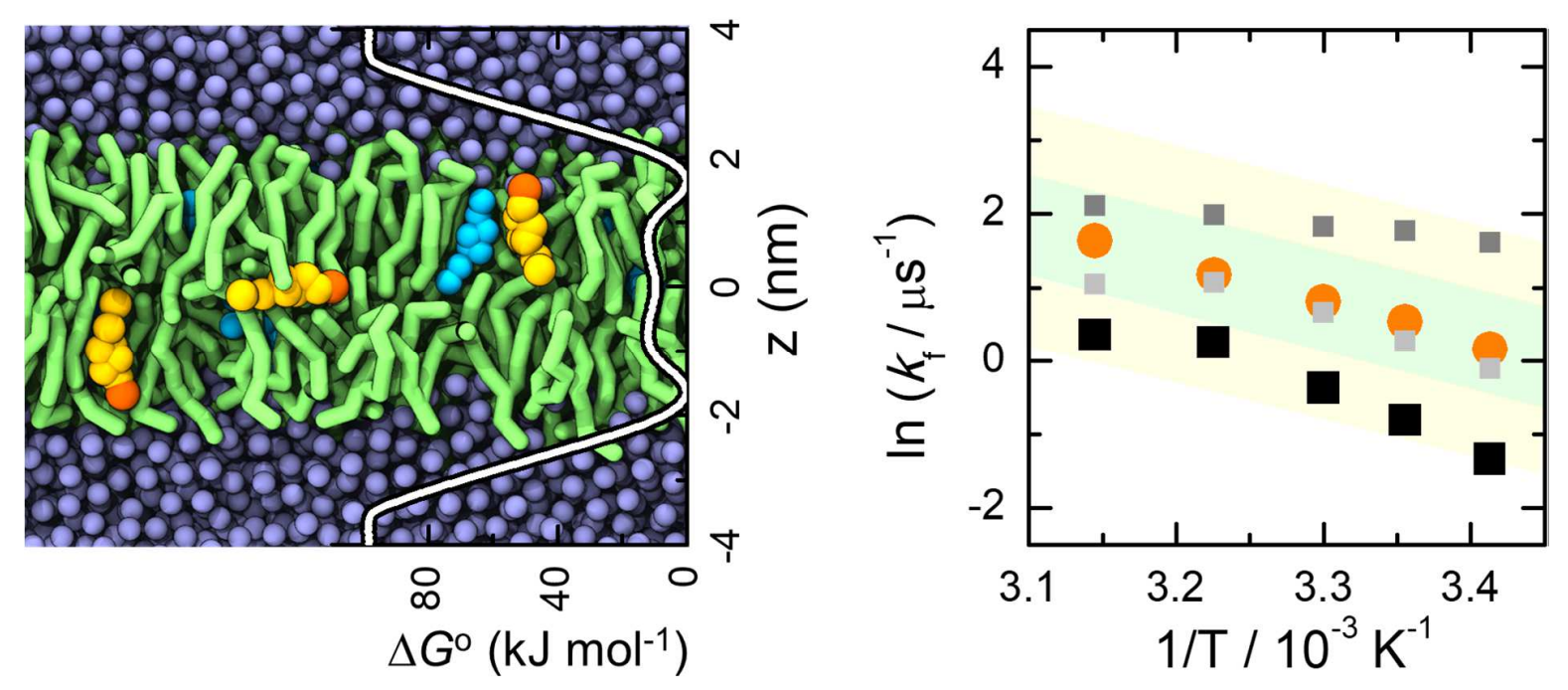